\title{
ON (APPROXIMATE) HOMOLOGICAL NOTIONS OF CERTAIN BANACH ALGEBRAS
}

\author{
AMIR SAHAMI ${ }^{*}$, ROSHANAK LOTFIKAR ${ }^{1}$, AND \\ AHMAD SOLTANMOHAMADI ${ }^{2}$
}

\author{
${ }^{1}$ Department of Mathematics Faculty of Basic Sciences Ilam University \\ P.O. Box 69315-516 Ilam, Iran.
}

${ }^{2}$ Faculty of Mathematics and Computer Science, Amirkabir University of Technology, $424 \mathrm{Hafez}$ Avenue, 15914 Tehran, Iran

*Corresponding author. E-mail: a.sahami@ilam.ac.ir

DOI: $10.20948 /$ mathmontis-2020-47-1

Summary. In this paper, we study the notion of $\phi$-biflatness, $\phi$-biprojectivity, approximate biprojectivity and Johnson pseudo-contractibility for a new class of Banach algebras. Using this class of Banach algebras, we give some examples which are approximately biprojective. Also some Banach algebras are given among matrix algebras which are never Johnson pseudo-contractible.

\section{INTRODUCTION}

Given a Banach algebra $A$, Kamyabi-Gol et al. in [4] defined a new product on $A$ which is denoted by $*$ In fact $a * b=a e b$, for each $a, b \in A$ where $e$ is an element of the closed unit ball $\overline{B_{1}^{0}}$ of $A$. A Banach algebra $A$ equipped with $*$ as its product is denoted by $A_{e}$. They studied some properties like amenability and Arens regularity of $A_{e}$. In [6] some homological properties of $A_{e}$ like biflatness, biprojectivity and $\phi$-amenability discussed.

New notions of $\phi$-amenability and approximate notions of homological Banach theory introduced and studied for Banach algebras see[14], [15] and [5]. In fact a Banach algebra a Banach algebra $A$ is called approximate $\phi$-contractible if there exists a net $\left(m_{\alpha}\right)$ in $A$ such that $a m_{\alpha}-\phi(a) m_{\alpha} \rightarrow 0$. and $\phi\left(m_{\alpha}\right)=1$, for every $a \in A$. where $\phi$ is a multiplicative linear functional on $A$. For more information see [2]. Also a Banach algebra $A$ is called approximate biprojective if there exists a net of bounded linear maps from $A$ into $A \otimes_{p} A$, say $\left(\rho_{\alpha}\right)_{\alpha \in I}$, such that

$$
\begin{aligned}
& \text { 1. } a \cdot \rho_{\alpha}(b)-\rho_{\alpha}(a b) \stackrel{\|\cdot\|}{\rightarrow} 0, \\
& \text { 2. } \rho_{\alpha}(b a)-\rho_{\alpha}(b) \cdot a \stackrel{\|\cdot\|}{\rightarrow} 0, \\
& \text { 3. } \pi_{A} \circ \rho_{\alpha}(a)-a \rightarrow 0,
\end{aligned}
$$

for every $a, b \in A$. In [1] the structure of approximate biprojective Banach algebras and its nilpotent ideals and also the relation with other notions of amenability are discussed.

We present some standard notations and definitions that we shall need in this paper. Let $A$ be a Banach algebra. Throughout this work, the character space of $A$ is denoted by $\Delta(A)$, that

2010 Mathematics Subject Classification: 46M10, 46H20, 46H05.

Key words and Phrases: Approximate $\phi$-contratiblity, Approximate biprojectivity, $\phi$-biflatness, $\phi$-biprojectivity, Johnson pseudo-contractibility, $\phi$-biflatness, Banach algebra. 
is, all non-zero multiplicative linear functionals on $A$. For each $\phi \in \Delta(A)$ there exists a unique extension $\tilde{\phi}$ to $A^{* *}$ which is defined $\tilde{\phi}(F)=F(\phi)$. It is easy to see that $\tilde{\phi} \in \Delta\left(A^{* *}\right)$. The projective tensor product $A \bigotimes_{p} A$ is a Banach $A$-bimodule via the following actions

$$
a \cdot(b \otimes c)=a b \otimes c .(b \otimes c) \cdot a=b \otimes c a \quad(a . b . c \in A) .
$$

The product morphism $\pi_{A}: A \bigotimes_{p} A \rightarrow A$ is given by $\pi_{A}(a \otimes b)=a b$, for every $a . b \in A$. Let $A$ and $B$ be Banach algebras. We denote by $\phi \otimes \psi$ a map defined by $\phi \otimes \psi(a \otimes b)=$ $\phi(a) \psi(b)$ for all $a \in A$ and $b \in B$. It is easy to see that $\phi \otimes \psi \in \Delta\left(A \otimes_{p} B\right)$.

Let $X$ and $Y$ be Banach $A$-bimodules. The map $T: X \rightarrow Y$ is called $A$-bimodule morphism, if

$$
T(a \cdot x)=a \cdot T(x) . \quad T(x \cdot a)=T(x) \cdot a . \quad(a \in A, x \in X) .
$$

Also a net of $\left(T_{\alpha}\right)$ of maps from $X$ into $Y$ is called approximate $A$-bimodule morphism, if

$$
T_{\alpha}(a \cdot x)-a \cdot T_{\alpha}(x) \rightarrow 0 . \quad T_{\alpha}(x \cdot a)-T_{\alpha}(x) \cdot a \rightarrow 0 . \quad(a \in A . x \in X) .
$$

The content of the paper is as follows. In section 2 we study $\phi$-homological properties of $A_{e}$ like $\phi$-biflatness and $\phi$-biprojectivity. Approximate biprojectivity and Johnson pseudocontractibility are two important notions of Banach homology theory, which we discuss for $A_{e}$ in section 3. We give some examples of matrix algebras to illustrate the paper.

\section{2. $\phi$ - HOMOLOGICAL PROPERTIES OF CERTAIN BANACH ALGEBRAS}

This section is devoted to the concepts of Banach homology related to a character $\phi$.

Proposition 2.1 [4, Proposition 2.3] Let $A$ be a Banach algebra and $e \in \overline{B_{1}^{0}}$. Then $A_{e}$ is unital if and only if $A$ is unital and $e$ is invertible.

Proposition 2.2 [4, Proposition 2.4] Let $A$ be a Banach algebra and $e \in \overline{B_{1}^{0}}$.Then the followings hold:

1. If $\phi$ is a multiplicative linear functional on $\mathrm{A}$, then $\phi(e) \phi$ is a multiplicative linear functional on $A_{e}$.

2. If $A_{e}$ is unital and $\psi$ is a multiplicative linear functional on $A_{e}$, then $\phi(a)=\psi\left(e^{-1} a\right)$ is a multiplicative linear functional on $\mathrm{A}$.

Proposition 2.3 [6, Proposition 2.3] Let $A$ be a Banach algebra and $e \in \overline{B_{1}^{0}}$. If $A_{e}$ is unital then $\left(A_{e}\right)_{e^{-2}}=A$, ( isometrically isomorphism ).

Proposition 2.4 Suppose that $A$ is a Banach algebra and also suppose that $e \in \overline{B_{1}^{0}}$ and $\phi \in \Delta(A)$. Then the followings hold:

1. If $A$ is approximate $\phi$-contractible and $\phi(e) \neq 0$, then $A_{e}$ is approximately $\psi$ contractible, where $\psi=\phi(e) \phi$.

2. If $A_{e}$ is unital and approximate $\psi$-contractible, then $A$ is approximate $\phi$-contractible, where $\phi(a)=\psi\left(e^{-1} a\right)$ for each $a \in A$.

Proof. Suppose that $A$ is approximately $\phi$-contractible. So there is a net $\left(m_{\alpha}\right)$ in $A$ such that 


$$
a m_{\alpha}-\phi(a) m_{\alpha} \rightarrow 0 . \quad \phi\left(m_{\alpha}\right)=1 . \quad(a \in A) .
$$

Define $n_{\alpha}=\frac{m_{\alpha}}{\phi(e)}$. Since $\psi(a)=\phi(a e)=\phi(e a)$, we have

$$
\begin{aligned}
a * n_{\alpha}-\psi(a) n_{\alpha} & =a e n_{\alpha}-\psi(a) n_{\alpha} \\
& =a e \frac{m_{\alpha}}{\phi(e)}-\psi(a) \frac{m_{\alpha}}{\phi(e)} \\
& =a e \frac{m_{\alpha}}{\phi(e)}-\phi(a e) \frac{m_{\alpha}}{\phi(e)}+\phi(a e) \frac{m_{\alpha}}{\phi(e)}-\psi(a) \frac{m_{\alpha}}{\phi(e)} \rightarrow 0 . \quad\left(a \in A_{e}\right) .
\end{aligned}
$$

Also

$$
\psi\left(n_{\alpha}\right)=\psi\left(\frac{m_{\alpha}}{\phi(e)}\right)=\phi(e) \phi\left(\frac{m_{\alpha}}{\phi(e)}\right)=\phi\left(m_{\alpha}\right)=1 .
$$

It follows that $A_{e}$ is approximate $\phi$-contractible.

Suppose that $\phi(a)=\psi\left(e^{-1} a\right)$ and also suppose that $A_{e}$ is unital and approximately left $\psi$-contractible. It is easy to see that $\psi(a)=\phi(e a)$. Let $\left(m_{\alpha}\right)$ be a net in $A_{e}$ such that

$$
a * m_{\alpha}-\psi(a) m_{\alpha} \rightarrow 0 . \quad \psi\left(m_{\alpha}\right)=1 . \quad\left(a \in A_{e}\right) .
$$

Since

$$
\begin{aligned}
a * m_{\alpha}-\psi(a) m_{\alpha} & =\operatorname{aem}_{\alpha}-\psi(a) m_{\alpha} \\
& =\operatorname{aem}_{\alpha}-\phi(e a) m_{\alpha} \\
& =\operatorname{aem}_{\alpha}-\phi(e) \phi(a) m_{\alpha} \\
& =\operatorname{aem}_{\alpha}-\phi(a) \phi(e) m_{\alpha} \\
& =\operatorname{aem}_{\alpha}-\phi(a e) m_{\alpha}
\end{aligned}
$$

we have

$$
a * m_{\alpha}-\psi(a) m_{\alpha}=a e m_{\alpha}-\phi(a e) m_{\alpha} \rightarrow 0
$$

for each $a \in A$. Replacing $a$ with $a e^{-1}$ we have $a m_{\alpha}-\phi(a) m_{\alpha} \rightarrow 0$. Regarding

$$
1=\psi\left(m_{\alpha}\right)=\phi\left(e m_{\alpha}\right)=\phi(e) \phi\left(m_{\alpha}\right) .
$$

we may suppose that $\phi\left(m_{\alpha}\right) \neq 0$, for each $\alpha$. Now define $n_{\alpha}=\frac{m_{\alpha}}{\phi\left(m_{\alpha}\right)}$. Clearly $\phi\left(n_{\alpha}\right)=1$. Also

$$
a n_{\alpha}-\phi(a) n_{\alpha}=a \frac{m_{\alpha}}{\phi\left(m_{\alpha}\right)}-\phi(a) \frac{m_{\alpha}}{\phi\left(m_{\alpha}\right)} \rightarrow 0
$$

It finishes the proof.

Example 2.5 In this example we show that there exists a Banach algebra $A_{e}$ which is not approximate $\psi$-contractible. Let $A=\left\{\left(\begin{array}{lll}a_{11} & a_{12} & a_{13} \\ 0 & a_{22} & a_{23} \\ 0 & 0 & a_{33}\end{array}\right) \mid a_{i j} \in \mathbb{C}\right\}$ and suppose that $e=$ 
$\left(\begin{array}{ccc}\frac{1}{6} & \frac{1}{6} & \frac{1}{6} \\ 0 & \frac{1}{6} & \frac{1}{6} \\ 0 & 0 & \frac{1}{6}\end{array}\right)$. Clearly A with matrix operations and $\ell^{1}$-norm is a Banach algebra. We know

that $e$ is invertible and by Proposition 2.1, $A_{e}$ is unital. Define $\phi: A \rightarrow \mathbb{C}$ by

$$
\phi\left(\left(\begin{array}{lll}
a_{11} & a_{12} & a_{13} \\
0 & a_{22} & a_{23} \\
0 & 0 & a_{33}
\end{array}\right)\right)=a_{33} .
$$

Clearly $\phi$ is a character(multiplicative linear functional) and $\phi(e) \neq 0$. Suppose conversely that $A_{e}$ is approximate $\psi$-contractible. By previous Proposition(2), $A$ becomes approximate $\phi$-contractible. On the other hand by the same arguments as in the proof of [7, Theorem 5.1] $A$ is not approximate $\phi$-contractible, which is a contradiction.

Let $A$ be a Banach algebra and $\phi \in \Delta(A) . A$ is called $\phi$-biprojective, if there exists a bounded $A$-bimodule morphism $\rho: A \rightarrow A \bigotimes_{p} A$ such that $\phi \circ \pi_{A} \circ \rho=\phi$. Also $A$ is called $\phi$ biflat if there exists a bounded $A$-bimodule morphism $\rho: A \rightarrow\left(A \bigotimes_{p} A\right)^{* *}$ such that $\tilde{\phi} \circ \pi_{A}^{* *} \circ$ $\rho=\phi$. For more information about $\phi$-biflatness and $\phi$-biprojectivity, the reader refers to [8] and [9].

Theorem 2.6 Let $A$ be a Banach algebra and $\phi \in \Delta(A)$. Suppose that $e \in \overline{B_{1}^{0}}$ and $\phi(e) \neq 0$. If $A$ is $\phi$-biprojective, then $A_{e}$ is $\psi=\phi(e) \phi$-biprojective.

Proof. Since $A$ is $\phi$-biprojective, there exists a bounded $A$-bimodule morphism $\rho: A \rightarrow$ $A \bigotimes_{p} A$ such that $\phi \circ \pi_{A} \circ \rho=\phi$. Define $\tilde{\rho}=\frac{1}{\phi(e)} \rho$. We show that $\tilde{\rho}$ is a bounded $A_{e^{-}}$ bimodule morphism. To see this, consider

$$
\begin{aligned}
\tilde{\rho}(a * b)=\frac{1}{\phi(e)} \rho(a * b)=\frac{1}{\phi(e)} \rho(a e b) & =a e \frac{1}{\phi(e)} \rho(b) \\
& =a * \frac{1}{\phi(e)} \rho(b) \\
& =a * \tilde{\rho}(b) . \quad\left(a . b \in A_{e}\right) .
\end{aligned}
$$

Also

$$
\begin{aligned}
\tilde{\rho}(a * b)=\frac{1}{\phi(e)} \rho(a * b)=\frac{1}{\phi(e)} \rho(a e b) & =\frac{1}{\phi(e)} \rho(a) b e \\
& =\frac{1}{\phi(e)} \rho(a) * b \\
& =\tilde{\rho}(a) * b . \quad\left(a . b \in A_{e}\right) .
\end{aligned}
$$

On the other hand, since

$$
\psi \circ \pi_{A_{e}} \circ \tilde{\rho}=\phi(e) \phi \circ \pi_{A} \circ \rho .
$$

we have

$$
\psi \circ \pi_{A_{e}} \circ \tilde{\rho}(a)=\phi(e) \phi \circ \pi_{A} \circ \rho(a)=\phi(e) \phi(a)=\psi(a) . \quad\left(a \in A_{e}\right) .
$$


So $A_{e}$ is $\psi$-biprojective.

Using the similar arguments as in the proof of the previous theorem, we have the following corollary:

Corollary 2.7 Let $A$ be a Banach algebra and $\phi \in \Delta(A)$. Suppose that $e \in \overline{B_{1}^{0}}$ and $\phi(e) \neq 0$. If $A$ is $\phi$-biflat, then $A_{e}$ is $\psi=\phi(e) \phi$-biflat.

Let $A$ be a Banach algebra and $\phi \in \Delta(A)$. $A$ is called $\phi$-amenable if there exists a bounded net $\left(m_{\alpha}\right)$ in $A$ such that $a m_{\alpha}-\phi(a) m_{\alpha} \rightarrow 0$ and $\phi\left(m_{\alpha}\right)=1$, for every $a \in A$. see [5].

Corollary 2.8 Let $A$ be a Banach algebra and $\phi \in \Delta(A)$. Suppose that $e \in \overline{B_{1}^{0}}$ and $\phi(e) \neq 0$. If $A$ is $\phi$-biflat and $A$ has a left approximate identity, then $A_{e}$ is approximate $\psi=\phi(e) \phi$ contractible.

Proof. Since $A$ is $\phi$-biflat and $A$ has a left approximate identity, by similar arguments as in the proof of [7, Theorem 2.2] $A$ is $\phi$-amenable. It is easy to see that $\phi$-amenability of $A$ implies that $A$ is approximate $\phi$-contractible. Applying Proposition 2.4, $A_{e}$ becomes approximate $\psi$-contractible.

Let $A \mathrm{~b}$ a Banach algebra and $\phi \in \Delta(A)$. Then $A$ is called approximate left $\phi$-biprojective if there exists a net of bounded linear maps from $A$ into $A \otimes_{p} A$, say $\left(\rho_{\alpha}\right)_{\alpha \in I}$, such that

$$
\begin{aligned}
& \text { 1. } \rho_{\alpha}(a b)-\phi(a) \rho_{\alpha}(b) \stackrel{\|\cdot\|}{\rightarrow} 0, \\
& \text { 2. } \rho_{\alpha}(b a)-\rho_{\alpha}(b) \cdot a \stackrel{\|\cdot\|}{\rightarrow} 0, \\
& \text { 3. } \pi_{A} \circ \rho_{\alpha}(a)-a \rightarrow 0,
\end{aligned}
$$

for every $a, b \in A$, see [12].

Theorem 2.9 Let $A$ be a Banach algebra and $\phi \in \Delta(A)$. Suppose that $e \in \overline{B_{1}^{0}}$ and $\phi(e) \neq 0$. If $A$ is approximate left $\phi$-biprojective, then $A_{e}$ is approximate left $\psi=\phi(e) \phi$-biprojective.

Proof. Since $A$ is approximate left $\phi$-biprojective, there exists a net of bounded linear maps $\left(\rho_{\alpha}\right)$ from $A$ into $A \otimes_{p} A$ such that

$$
\rho_{\alpha}(a b)-\phi(a) \rho_{\alpha}(b) \rightarrow 0, \quad \rho_{\alpha}(a b)-\rho_{\alpha}(a) \cdot b \rightarrow 0, \quad \phi \circ \pi_{A} \circ \rho(a)-\phi(a) \rightarrow 0 .
$$

Define $\tilde{\rho}_{\alpha}=\frac{1}{\phi(e)} \rho_{\alpha}$. We show that there exists a net of bounded linear maps $\left(\tilde{\rho}_{\alpha}\right)$ from $A_{e}$ in to $A_{e} \otimes_{p} A_{e}$ such that

$$
\tilde{\rho}_{\alpha}(a * b)-\psi(a) \tilde{\rho}_{\alpha}(b) \rightarrow 0 . \quad \tilde{\rho}_{\alpha}(a * b)-\tilde{\rho}_{\alpha}(a) * b \rightarrow 0 . \quad \psi \circ \pi_{A} \circ \tilde{\rho}(a)-\psi(a) \rightarrow 0 .
$$

To see this, consider

$$
\begin{aligned}
\tilde{\rho}_{\alpha}(a * b)-\psi(a) \tilde{\rho}_{\alpha}(b) & =\tilde{\rho}_{\alpha}(a e b)-\phi(a) \phi(e) \tilde{\rho}_{\alpha}(b) \\
& =\frac{1}{\phi(e)}\left(\rho_{\alpha}(a e b)-\phi(a) \phi(e) \rho_{\alpha}(b)\right) \\
& =\frac{1}{\phi(e)}\left(\rho_{\alpha}(a e b)-\phi(a e) \rho_{\alpha}(b)+\phi(a e) \rho_{\alpha}(b)-\phi(a) \phi(e) \rho_{\alpha}(b)\right) \\
& \rightarrow 0
\end{aligned}
$$


Also

$$
\tilde{\rho}_{\alpha}(a * b)-\tilde{\rho}_{\alpha}(a) * b=\frac{1}{\phi(e)} \rho_{\alpha}(a e b)-\frac{1}{\phi(e)} \rho_{\alpha}(a) e b \rightarrow 0
$$

On the other hand, since

$$
\psi \circ \pi_{A_{e}} \circ \tilde{\rho}_{\alpha}=\phi(e) \phi \circ \pi_{A} \circ \rho_{\alpha}
$$

we have for $\left(a \in A_{e}\right.$

$$
\psi \circ \pi_{A_{e}} \circ \tilde{\rho}_{\alpha}(a)-\psi(a)=\phi(e) \phi \circ \pi_{A} \circ \rho_{\alpha}(a)-\phi(e) \phi(a) \rightarrow \phi(e) \phi(a)-\phi(e) \phi(a)=0 .
$$

So $A_{e}$ is approximate left $\psi$-biprojective.

Remark 2.10 Let $A$ and $B$ be Banach algebras and $e_{A} \in{\overline{B_{1}^{0}}}^{A}$ and $e_{B} \in{\overline{B_{1}^{0}}}^{B}$. Then there exist two sequences $\left(x_{n}\right)$ and $\left(y_{n}\right)$ in the unit ball $A$ and the unit ball $B$ such that $x_{n} \rightarrow e_{A}$ and $y_{n} \rightarrow e_{B}$. respectively. Since

$$
\left\|x_{n} \otimes y_{n}-e_{A} \otimes e_{B}\right\| \leq\left\|x_{n} \otimes y_{n}-e_{A} \otimes y_{n}\right\|+\left\|e_{A} \otimes y_{n}-e_{A} \otimes e_{B}\right\| \rightarrow 0 .
$$

we have $e_{A} \otimes e_{B} \in{\overline{B_{1}^{0}}}^{A \otimes_{p} B}$. Define $T: A_{e_{A}} \otimes_{p} B_{e_{B}} \rightarrow A \otimes_{p} B_{e_{A} \otimes e_{B}}$ by $T(a \otimes b)=a \otimes b$ for every $a \in A$ and $b \in B$. It is easy to see that $T$ is an isometric algebra isomorphism. Also $T$ is a bounded $A \otimes_{p} B_{e_{A} \otimes e_{B}}$-bimodule morphism.

Proposition 2.11 Let $A$ and $B$ be Banach algebras and $e_{A} \in{\overline{B_{1}^{0}}}^{A}$ and $e_{B} \in{\overline{B_{1}^{0}}}^{B}$. Suppose that $\phi_{A} \in \Delta(A)$ and $\phi_{B} \in \Delta(B)$ which $\phi_{A}\left(e_{A}\right) \neq 0$ and $\phi_{B}\left(e_{B}\right) \neq 0$. If $A$ and $B$ are $\phi_{A}$-biprojective and $\phi_{B}$-biprojective, respectively, then $A \otimes_{p} B_{e_{A} \otimes e_{B}}$ is $\phi_{A}\left(e_{A}\right) \phi_{A} \otimes$ $\phi_{B}\left(e_{B}\right) \phi_{B}$-biprojective.

Proof. Since $A$ and $B$ are $\phi_{A}$-biprojective and $\phi_{B}$-biprojective, respectively, then by Theorem 2.9, $A_{e}$ and $B_{e}$ are $\phi_{A}\left(e_{A}\right) \phi_{A}$-biprojective and $\phi_{B}\left(e_{B}\right) \phi_{B}$-biprojective, respectively. So there exist a $A_{e_{A}}$-bimodule morphism $\rho_{0}: A_{e_{A}} \rightarrow A_{e_{A}} \otimes_{p} A_{e_{A}}$ and a $B_{e_{B}}{ }^{-}$ bimodule morphism $\rho_{1}: B_{e_{B}} \rightarrow B_{e_{B}} \otimes_{p} B_{e_{B}}$ such that $\phi_{A}\left(e_{A}\right) \phi_{A} \circ \pi_{A} \circ \rho_{0}=\phi_{A}\left(e_{A}\right) \phi_{A}$ and $\phi_{B}\left(e_{B}\right) \phi_{B} \circ \pi_{B} \circ \rho_{1}=\phi_{B}\left(e_{B}\right) \phi_{B}$.

Define $\theta:\left(A_{e_{A}} \otimes_{p} A_{e_{A}}\right) \otimes_{p}\left(B_{e_{B}} \otimes_{p} B_{e_{B}}\right) \rightarrow\left(A_{e_{A}} \otimes_{p} B_{e_{B}}\right) \otimes_{p}\left(A_{e_{A}} \otimes_{p} B_{e_{B}}\right)$ by

$$
\left(a_{1} \otimes a_{2}\right) \otimes\left(b_{1} \otimes b_{2}\right) \mapsto\left(a_{1} \otimes b_{1}\right) \otimes\left(a_{2} \otimes b_{2}\right) .
$$

where $a_{1} . a_{2} \in A$ and $b_{1} . b_{2} \in B$. Clearly $\theta$ is an isometric algebra isomorphism. Set $\rho=$ $(T \otimes T) \circ \theta \circ\left(\rho_{0} \otimes \rho_{1}\right) \circ T^{-1}$, where $T$ is the map defined as in Remark 2.10. We know that $\rho$ is a bounded linear map from $A \otimes_{p} B_{e_{A} \otimes e_{B}}$ into $\left(A \otimes_{p} B_{e_{A} \otimes e_{B}}\right) \bigotimes_{p}\left(A \otimes_{p} B_{e_{A} \otimes e_{B}}\right)$. Consider 


$$
\begin{aligned}
& \pi_{A \otimes_{p} B_{e_{A} \otimes e_{B}}} \circ \theta\left(a_{1} \otimes a_{2} \otimes b_{1} \otimes b_{2}\right)=\pi_{A \otimes_{p} B_{e_{A} \otimes e_{B}}}\left(a_{1} \otimes b_{1} \otimes a_{2} \otimes b_{2}\right) \\
& =\pi_{A_{e_{A}}}\left(a_{1} \otimes a_{2}\right) \otimes \pi_{B_{e_{B}}}\left(b_{1} \otimes b_{2}\right) \text {. }
\end{aligned}
$$

then clearly one can show that $\pi_{A \otimes_{p} B_{e_{A} \otimes e_{B}}} \circ \theta=\pi_{A_{e_{A}}} \otimes \pi_{B_{e_{B}}}$. Hence,

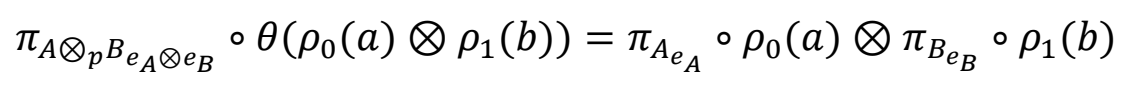

and it is easy to see that

$$
\phi_{A}\left(e_{A}\right) \phi_{A} \otimes \phi_{B}\left(e_{B}\right) \phi_{B} \circ \pi_{A \otimes_{p} B} \circ \theta\left(\rho_{0} \otimes \rho_{1}\right)(a \otimes b)=\phi_{A}\left(e_{A}\right) \phi_{A} \otimes \phi_{B}\left(e_{B}\right) \phi_{B}(a \otimes b) \text {. }
$$

the proof is complete.

\section{APPROXIMATE HOMOLOGICAL PROPERTIES OF CERTAIN BANACH ALGEBRAS}

In this section we investigate approximate biprojectivity and Johnson pseudocontractibility of $A_{e}$.

Theorem 3.1 Suppose that $A$ is a Banach algebra and also suppose that $e \in \overline{B_{1}^{0}}$. Then the followings hold:

1. If $A$ is approximately biprojective and $A_{e}$ is unital then $A_{e}$ is approximately biprojective.

2. If $A_{e}$ is unital and approximately biprojective, then $A$ is approximately biprojective.

Proof. To show (1), suppose that $A$ is approximately biprojective and $A_{e}$ is unital. It follows that there is an approximately $A$-bimodule morphism $\left(\rho_{\alpha}\right)$ from $A$ into $A \otimes_{p} A$ such that $\pi_{A} \circ \rho_{\alpha}(a)-a \rightarrow 0$ for each $a \in A$. Note that

$$
\begin{aligned}
\rho_{\alpha}(a * b)-a * \rho_{\alpha}(b) & =\rho_{\alpha}(a e b)-a * \rho_{\alpha}(b) \\
& =\rho_{\alpha}(a e b)-a e \rho_{\alpha}(b)+a e \rho_{\alpha}(b)-a * \rho_{\alpha}(b) \rightarrow 0 .
\end{aligned}
$$

and

$$
\begin{aligned}
\rho_{\alpha}(a * b)-\rho_{\alpha}(a) * b & =\rho_{\alpha}(a e b)-\rho_{\alpha}(a) * b \\
& =\rho_{\alpha}(a e b)-\rho_{\alpha}(a) e b+\rho_{\alpha}(a) e b-\rho_{\alpha}(a) * b \rightarrow 0,
\end{aligned}
$$

for each $a \in A_{e}$. It implies that $\left(\rho_{\alpha}\right)$ from $A_{e}$ into $A_{e} \otimes_{p} A_{e}$ is an approximately $A_{e}$-bimodule morphism. Define $T: A_{e} \otimes_{p} A_{e} \rightarrow A_{e} \otimes_{p} A_{e}$ by $T(a \otimes b)=a e^{-1} \otimes b$. Note that using Proposition 2.1, the definition of $T$ makes sense. It is easy to see that

$$
T(a *(c \otimes d))=a * T(c \otimes d) . \quad T((c \otimes d) * a)=T(c \otimes d) * a . \quad(\text { a.c. } d \in A) .
$$

Set $\tilde{\rho}_{\alpha}=T \circ \rho_{\alpha}$. Using direct calculations we can see that

$$
\pi_{A_{e}} \circ \tilde{\rho}_{\alpha}=\pi_{A} \circ \rho_{\alpha}
$$

It follows that $\pi_{A_{e}} \circ \tilde{\rho}_{\alpha}-a=\pi_{A} \circ \rho_{\alpha}-a \rightarrow 0 . \quad\left(a \in A_{e}\right)$.

Thus $A_{e}$ is approximately biprojective. 
To show (2), suppose that $A_{e}$ is unital and approximately biprojective. By Proposition 2.3, we know that $A=\left(A_{e}\right)_{e^{-2}}$. Now applying (1) it is easy to see that $A$ is approximately biprojective.

A Banach algebra $A$ is called biprojective if there exists a bounded $A$-bimodule morphism $\rho: A \rightarrow A \otimes_{p} A$ such that $\pi_{A} \circ \rho(a)=a$ for each $a \in A$. see [13].

Example 3.2 In this example we give a Banach algbra $A_{e}$ which is approximately biprojective. Let $A=\left\{\left(\begin{array}{lll}a_{11} & a_{12} & a_{13} \\ a_{21} & a_{22} & a_{23} \\ a_{31} & a_{32} & a_{33}\end{array}\right) \mid a_{i j} \in \mathbb{C}\right\}$. With the matrix operations and $\ell^{1}$-norm, A becomes a Banach algebra. Suppose that $e=\left(\begin{array}{ccc}\frac{1}{4} & \frac{1}{4} & 0 \\ 0 & \frac{1}{4} & 0 \\ 0 & 0 & \frac{1}{4}\end{array}\right)$. Clearly e is invertible and $A$ is unital. So by Proposition 2.1, $A_{e}$ is unital. It is well-known that $A$ is biprojective, see [13]. So $A$ is approximately biprojective. Applying previous theorem $A_{e}$ becomes approximately biprojective.

Definition 3.3 We say that a Banach algebra $A$ has approximate $(F)$-property (or $A$ is $A F P)$ if there is an approximate $A$-bimodule morphsim $\left(\rho_{\alpha}\right)$ from $A$ into $\left(A \bigotimes_{p} A\right)^{* *}$ such that $\pi_{A}^{* *} \circ \rho_{\alpha}(a)-a \rightarrow 0$. for each $a \in A$.

For the motivation of this definition see [3].

Proposition 3.4 If $A$ is $A F P$ and $A_{e}$ is unital, then $A_{e}$ is approximately biprojective.

Proof. Since $A$ is AFP, there exists an approximate $A$-bimodule morphsim $\left(\rho_{\alpha}\right)$ from $A$ into $\left(A \otimes_{p} A\right)^{* *}$ such that $\pi_{A}^{* *} \circ \rho_{\alpha}(a)-a \rightarrow 0$, for each $a \in A$. It is easy to see that $\left(\rho_{\alpha}\right)$ is an approximate $A_{e}$-bimodule morphsim from $A_{e}$ into $\left(A_{e} \otimes_{p} A_{e}\right)^{* *}$ such that $\pi_{A_{e}}^{* *} \circ \rho_{\alpha}(a)-$ $a \rightarrow 0$. for each $a \in A_{e}$. Let $T: A_{e} \otimes_{p} A_{e} \rightarrow A_{e} \bigotimes_{p} A_{e}$ be the same map as in the proof of Theorem 3.1. Clearly $T$ is $A_{e}$-module morphism, so is $T^{* *}$. Similar to the proof of Theorem 3.1, for the net $\left(T^{* *} \circ \rho_{\alpha}\right)$ is an approximate $A_{e}$-bimodule morphism from $A_{e}$ into $\left(A_{e} \otimes_{p} A_{e}\right)^{* *}$ such that

$$
\pi_{A_{e}}^{* *} \circ T^{* *} \circ \rho_{\alpha}(a)-a=\pi_{A}^{* *} \circ \rho_{\alpha}(a)-a \rightarrow 0 . \quad(a \in A) .
$$

We denote the identity of $A_{e}$ with $a_{0}$ and define $m_{\alpha}=\rho_{\alpha}\left(a_{0}\right)$. Clearly $\left(m_{\alpha}\right)$ is a net in $\left(A_{e} \otimes_{p} A_{e}\right)^{* *}$ which satisfies

$$
a * m_{\alpha}-m_{\alpha} * a \rightarrow 0 . \quad \pi_{A_{e}}^{* *}\left(m_{\alpha}\right) * a-a \rightarrow 0 . \quad\left(a \in A_{e}\right) .
$$

Take $\epsilon>0$ and arbitrary finite subsets $F \subseteq A_{e}, \Lambda \subseteq\left(A_{e} \otimes_{p} A_{e}\right)^{*}$ and $\Gamma \subseteq A_{e}^{*}$. Then we have

$$
\left\|a * m_{\alpha}-m_{\alpha} * a\right\|<\epsilon . \quad\left\|\pi_{A_{e}}^{* *}\left(m_{\alpha}\right) * a-a\right\|<\epsilon . \quad(a \in F) .
$$


It is well-known that for each $\alpha$, there exists a net $\left(n_{\beta}^{\alpha}\right)_{\beta}$ in $A_{e} \bigotimes_{p} A_{e}$ such that $n_{\beta}^{\alpha} \stackrel{w^{*}}{\rightarrow} m_{\alpha}$. Since $\pi_{A_{e}}^{* *}$ is a $w^{*}$-continuous map, we have $\pi_{A_{e}}\left(n_{\beta}^{\alpha}\right)=\pi_{A_{e}}^{* *}\left(n_{\beta}^{\alpha}\right) \stackrel{w^{*}}{\rightarrow} \pi_{A}^{* *}\left(m_{\alpha}\right)$.

Thus we have $\left|a * n_{\beta}^{\alpha}(f)-a * m_{\alpha}(f)\right|<\frac{\epsilon}{K_{0}} . \quad\left|n_{\beta}^{\alpha} * a(f)-m_{\alpha} *(f)\right|<\frac{\epsilon}{K_{0}}$ and

$$
\left|\pi_{A_{e}}\left(n_{\beta}^{\alpha}\right)(g)-\pi_{A_{e}}^{* *}\left(m_{\alpha}\right)(g)\right|<\frac{\epsilon}{K_{1}} .
$$

for each $a \in F, f \in \Lambda$ and $g \in A^{*}$, where $K_{0}=\sup \{\|f\|: f \in \Lambda\}$ and $K_{1}=\sup \{\|g\|: g \in \Gamma\}$. Since $a * m_{\alpha}-m_{\alpha} * a \rightarrow 0$ and $\pi_{A_{e}}^{* *}\left(m_{\alpha}\right) * a-a \rightarrow 0$. we can find $\beta=\beta(F . \Lambda$. Г. $\epsilon)$ such that

$$
\left|a * n_{\beta}^{\alpha}(f)-n_{\beta}^{\alpha} * a(f)\right|<c \frac{\epsilon}{K_{0}} \quad\left|\pi_{A_{e}}\left(n_{\beta}^{\alpha}\right) * a(g)-a(g)\right|<\frac{\epsilon}{K_{1}} . \quad(a \in F . f \in \Lambda . g \in \Gamma)
$$

for some $c \in \mathbb{R}^{+}$. Using Mazur's lemma, we have a net $\left(n_{(F . \Lambda . \Gamma . \epsilon)}\right)$ in $A_{e} \otimes_{p} A_{e}$ such that

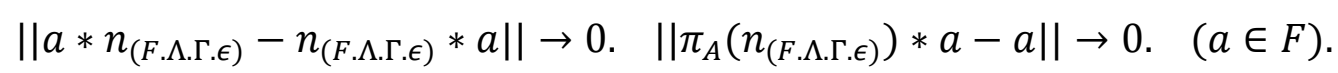

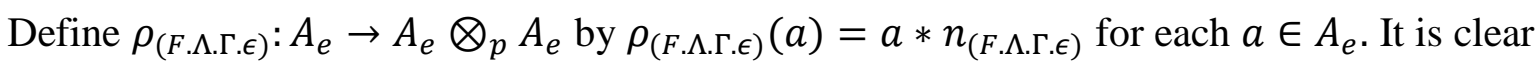
that $\rho_{(F . \Lambda . \Gamma . \epsilon)}(a * b)=a * \rho_{(F . \Lambda . \Gamma . \epsilon)}(b)$ for each $a, b \in A$. Also

$$
\begin{aligned}
\left\|\rho_{(F . \Lambda . \epsilon)}(a * b)-\rho_{(F . \Lambda . \Gamma . \epsilon)}(a) * b\right\| & =\left\|a b * n_{(F . \Lambda . \Gamma . \epsilon)}-a *\left(n_{(F . \Lambda . \Gamma . \epsilon)} * b\right)\right\| \\
& \leq\|a\|\left\|b * n_{(F . \Lambda . \Gamma . \epsilon)}-n_{(F . \Lambda . \Gamma . \epsilon)} * b\right\| \rightarrow 0 .
\end{aligned}
$$

for each $a . b \in A_{e}$. Also

$$
\begin{aligned}
\left\|\pi_{A_{e}} \circ \rho_{(F . \Lambda . \Gamma . \epsilon)}(a)-a\right\| & =\left\|\pi_{A_{e}}\left(a * n_{(F . \Lambda . \Gamma . \epsilon)}\right)-a\right\| \\
& =\left\|\pi_{A_{e}}\left(a * n_{(F . \Lambda . \Gamma . \epsilon)}\right)-\pi_{A_{e}}\left(n_{(F . \Lambda . \Gamma . \epsilon)} * a\right)+\pi_{A_{e}}\left(n_{(F . \Lambda . \Gamma . \epsilon)} * a\right)-a\right\| \\
& \leq\left\|\pi_{A_{e}}\left(a * n_{(F . \Lambda . \Gamma . \epsilon)}\right)-\pi_{A_{e}}\left(n_{(F . \Lambda . \Gamma . \epsilon)} * a\right)\right\|+\left\|\pi_{A_{e}}\left(n_{(F . \Lambda . \Gamma . \epsilon)}\right) * a-a\right\| \\
& \rightarrow 0 .
\end{aligned}
$$

for each $a \in F$. Thus with respect to the net $\left(\rho_{(F . \Lambda . \Gamma . \epsilon)}\right)_{(F . \Lambda . \Gamma . \epsilon)}$. $A_{e}$ becomes approximately biprojective.

A Banach algebra $A$ is called Johnson pseudo-contractible, if there exists a not necessarily bounded net $\left(m_{\alpha}\right)$ in $\left(A \otimes_{p} A\right)^{* *}$ such that $a \cdot m_{\alpha}=m_{\alpha} \cdot a$ and $\pi_{A}^{* *}\left(m_{\alpha}\right) a-a \rightarrow 0$. for every $a \in A$, see [11] and [10].

A Banach algebra $A$ is called biflat, if there is a bounded $A$-bimodule morphsim $\rho$ from $A$ into $\left(A \otimes_{p} A\right)^{* *}$ such that $\pi_{A}^{* *} \circ \rho_{\alpha}(a)=a$, for each $a \in A$, see [13].

Proposition 3.5 Let $A$ be a Banach algebra and $e \in \overline{B_{1}^{0}}$. Suppose that $A_{e}$ is unital. Then $A$ is Johnson pseudo-contractible if and only if $A_{e}$ is Johnson pseudo-contractible.

Proof. Since $A_{e}$ is unital, by Proposition $2.1 A$ is unital. So using [3, Theorem 2.1], Johnson pseudo-contractibility of $A$ implies that $A$ is amenable. Thus by [13, Exercise 4.3.15], $A$ is biflat. Then by [6, Theorem 2.4] $A_{e}$ is biflat. Since $A_{e}$ is unital, biflatness of $A_{e}$ gives the amenability of $A_{e}$.

For converse, suppose that $A_{e}$ is Johnson pseudo-contractible. Since $A_{e}$ is unital by [3, Theorem 2.1] $A_{e}$ is amenable, so is biflat. Applying [6, Theorem 2.4] follows that $A$ is biflat. 
Using Proposition 2.1, $A$ is unital, thus by [13, Exercise 4.3.15] $A$ is amenable. So [11, Lemma 2.1] implies that $A$ is Johnson pseudo-contractible.

Example 3.6 We give a Banach algebra $A_{e}$ which is not Johnson pseudo-contractible. Let

$A=\left\{\left(\begin{array}{lll}a_{11} & a_{12} & a_{13} \\ 0 & a_{22} & a_{23} \\ 0 & 0 & a_{33}\end{array}\right) \mid a_{i j} \in \mathbb{C}\right\}$ and suppose that $e=\left(\begin{array}{lll}\frac{1}{4} & \frac{1}{4} & 0 \\ 0 & \frac{1}{4} & 0 \\ 0 & 0 & \frac{1}{4}\end{array}\right)$. Clearly $e$ is invertible

and $A$ is unital. So by Proposition $2.1 A_{e}$ is unital. Using [11, Theorem 2.5] we know that $A$ is not Johnson pseudo-contractible. So by previous proposition $A_{e}$ is not Johnson pseudocontractible.

\section{REFERENCES}

[1] H. P. Aghababa, "Approximately biprojective Banach algebras and nilpotent ideals". Bull. Aust. Math. Soc. 87, 1-12 (2012).

[2] H. P. Aghababa, L. Y. Shi and Y. J. Wu, "Generalized notions of character amenability". Acta Math. Sin, 29, 1329-1350 (2013).

[3] M. Askari-Sayah, A. Pourabbas and A. Sahami, "Johnson pseudo-contractibility of certain Banach algebras and its nilpotent ideals". Analysis Mathematica, 3, 461-473 (2019).

[4] R. A. Kamyabi-Gol and M. Janfada, "Banach algebras related to the elements of the unit ball of a Banach algebra.", Taiwan. J. Math., 12, 1769-1779 (2008).

[5] E. Kaniuth, A. T. Lau and J. Pym, "On $\phi$-amenability of Banach algebras", Math. Proc. Camb. Philos. Soc. 44, 85-96 (2008).

[6] A. R. Khoddami, " Biflatness, biprojectivity, $\phi$-amenability and $\phi$-contractibility of certain Banach algebras”, U.P.B. Sci. Bull., Series A, 80 (2), 81-86 (2018).

[7] A. Sahami, "On biflatness and $\phi$-biflatness of some Banach algebras", U.P.B. Sci. Bull., Series A, Vol. 80, Iss. 2, 112-123 (2018).

[8] A. Sahami and A. Pourabbas, "On $\phi$-biflat and $\phi$-biprojective Banach algebras", Bull. Belg. Math. Soc. Simon Stevin, 20, 789-801 (2013).

[9] A. Sahami and A. Pourabbas, "Approximate biprojectivity and $\phi$-biflatness of some Banach algebras", Colloq. Math, 145, 273-284 (2016).

[10] A. Sahami and A. Pourabbas, "Johnson pseudo-contractibility of various classes of Banach algebras", Bull. Belg. Math. Soc. Simon Stevin, 25, 171-182 (2018).

[11] A. Sahami and A. Pourabbas, "Johnson pseudo-contractibility of certain semigroup algebras", Semigroup Forum, 97, 203-207 (2018).

[12] A. Sahami and A. Pourabbas, "On approximate left $\phi$-biprojective Banach algebras", Glasnik Matematicki, Vol. 53, 187-203 (2018).

[13] V. Runde, Lectures on Amenability, Springer, New York, (2002).

[14] E. Samei, N. Spronk and R. Stokke, "Biflatness and pseudo-amenability of Segal algebras", Canad. J. Math. 62, 845-869 (2010).

[15] Y. Zhang, " Nilpotent ideals in a class of Banach algebras“, Proc. Amer. Math. Soc. 127, 32373242 (1999).

Received October 31, 2019 